\title{
Correlation between FIX genotype and pharmacokinetics of Nonacog alpha according to a multicentre Italian study
}

\author{
M. MORFINI, *A. DRAGANI, $\dagger$ E. PALADINO, + P. RADOSSI, $\mathbb{S}$ G. DI MINNO, \\ M. G. MAZZUCCONI,**G. ROSSETTI, $+\dagger$ G. BARILLARI, + * M. NAPOLITANO $\mathbb{S}$ and \\ G. TAGARIELLOS \\ *Past President of Italian Association of Haemophilia Centres (AICE), Firenze; †Haemophilia \& Thrombosis Centre, \\ Haematology Department, Regional Hospital, Pescara; $\$$ Atherothrombotic Diseases, Cardio-Vascular Department, University \\ Hospital of Florence, Florence; $\$$ Haematology and Haemophilia Centre, Regional Hospital, Castelfranco Veneto; $\llbracket$ Reference \\ Centre for Haemophilia and Thrombosis, Department of Clinical and Experimental Medicine Federico II University, Naples; \\ *Haemophilia \& Thrombosis Centre, Haematology Department, La Sapienza University, Rome; ††Haemophilia Centre \\ Santa Chiara Hospital, Trento; \$Haemophilia Centre, S. Maria della Misericordia Hospital, Udine; and \$\$Haemophilia ¿ \\ Thrombosis Centre, Haematology Department, University of Palermo, Palermo, Italy
}

\begin{abstract}
Introduction: Pharmacokinetic (PK) studies on recombinant FIX concentrate, Nonacog alpha, were conducted with different sampling time designs which gave rise to not complete and homogenous outcomes. In addition, patient's FIX genotype/PK relationship has never been investigated. Aim: Investigate how different sampling times may affect PK parameters and try to find a FIX genotype/PK relationship. Patients and Methods: A cohort pharmacokinetic, Nonacog Alpha single-dose, open-label, non-comparative study was conducted in eight Comprehensive Care Haemophilia Centres in Italy. Seventeen previously treated moderate or severe haemophilia B patients were enrolled. Factors IX:C one-stage clotting assay, FIX genotype and PK analysis were centralized. Results: The evaluation of PK outcomes showed a quite long half-life, smaller clearance and volume of distribution of Nonacog Alpha in comparison with the results from previously reported studies, where blood sampling was stopped too early. The relationship between PK outcomes and FIX genotype showed that small deletions displayed the higher clearance and shorter half-life, the nonsense mutations (the lower and the longer respectively), and missense mutations were in between. Conclusions: It is evident that area under the curve (AUC) and other PK parameters depend from the sampling time design. In order to have a complete evaluation of clotting factors in vivo decay, blood samples must be collected until the baseline factor concentration has been achieved again. Due to the relationship between FIX genotype and clearance, tailored prophylaxis of HB patients could be partially predicted by genotyping.
\end{abstract}

Keywords: DNA Recombinant proteins, factor IX, genotype, haemophilia B, pharmacokinetics

\section{Introduction}

Very few well-designed studies have been conducted to achieve complete information about the PK of rDNA derived factor IX concentrate, Nonacog Alpha,

Correspondence: Massimo Morfini, Past President of Italian Association of Haemophilia Centres (AICE), Via Statuto n.1, I-50129 Firenze, Italy.

Tel.: +39-348-2306928; fax: +39-055-473218;

e-mail: massimo.morfini@unifi.it

Accepted after revision 5 January 2016 in haemophilia B patients. In some studies, the sampling times or PK analysis were not adequate, sometimes limited to 48th hour, sometimes extended to 72 nd hour. In addition, the FIX genotype of patients was never reported.

Nonacog Alpha (BeneFix ${ }^{\mathrm{TM}}$, Pfizer Inc, New York, NY, USA), recombinant coagulation factor IX, is a 415-amino acid glycoprotein (approximately 55k.Da) produced in Chinese hamster ovary $(\mathrm{CHO})$ cells. It belongs to the so called 'Third generation recombinant concentrates' because the medium where the cells grow and the final formulation do not contain 
any animal or human protein. After two steps of purification by ion exchange and affinity chromatography, Nonacog alpha is submitted to nanofiltration (20 nm pores) in order to remove any pathogens, eventually present in the cell culture. The first article [1] published in 1997 was a report of the regulatory bioequivalence study conducted in a small cohort of patients $(n=11)$ comparing Nonacog alpha against a monoclonal plasma-derived FIX (pdFIX) concentrate. Only in vivo recovery (IVR), half-life (HL) and mean residence time (MRT) were reported. Another crossover study confirmed the low IVR of Benefix with respect to pdFIX even though HL was similar in both products [2]. A well-designed study on a large cohort of patients $(n=56)$ conducted at the switch from pdFIX concentrate to Nonacog alpha reported again similar outcomes, but again limited to IVR and HL [3]. The raw data of another previously published study [4] were afterwards analysed by S. Bjorkman in order to determine the standard pharmacokinetic parameters according to a one- and two-open compartment model: for the first time, also clearance $(\mathrm{Cl})$, volume of distribution at steady state (Vdss), and terminal half-life $(\mathrm{t} 1 / 2)$ were published further than IVR and MRT [5]. A new double-blind, randomized, pharmacokinetic crossover study involved the new reformulated Nonacog alpha in comparison with the original Nonacog Alpha [6]. Analysis of AUC, both percentage as well as incremental IVR, and terminal half-life did not show any difference between the two formulations. Most of these studies have been conducted with different and sometimes inadequate study designs. Only the analysis performed by S. Bjorkman [5] provided a complete report of all the PK parameters needed for a good evaluation of Nonacog alpha. For these reasons, we planned to conduct in Italy a multicentre PK study designed according to the ISTH recommendations $[7,8]$, taking into account also the FIX genotype of each patient.

The primary objective of the study was to investigate the variability in all PK parameters (in vivo recovery, AUC, clearance, MRT, elimination rate constant, volume of distribution, terminal half-life of Nonacog alpha and the correlation between FIX genotype and PK outcomes, in severe and moderate haemophilia B previously treated patients. The secondary objective was to evaluate different methods of PK analysis.

\section{Patients and methods}

Single-dose PK was performed in eight Comprehensive Care Haemophilia Centres (HCC) of Italy. Seventeen moderate and severe haemophilia B patients have been enrolled in the study. PK study was performed according to the ISTH recommendations: after a wash out of at least 7 days, a single dose, $47.70 \pm 4.13 \mathrm{IU} \mathrm{kg}^{-1}$ b.w., of Nonacog alpha was injected in less than $10 \mathrm{~min}$ and blood samples were collected just before the infusion (baseline FIX concentration) and $0.25,1$, $3,9,24,48$, and $72 \mathrm{~h}$ after the end of infusion. Three aliquots of platelet poor citrated plasma of each sample were snap frozen and stored at $-80^{\circ} \mathrm{C}$. The samples were shipped within 30 days, in dry ice to the central laboratory located in the Haemophilia Centre of Florence. FIX:C was assayed by means of one-stage clotting method, performed by means of coagulometer Behring Coagulation Time (BCT) of Siemens Healthcare Diagnostics using three double dilutions of each sample compared with a reference curve of standard human plasma (Siemens Healthcare GmbH, Erlangen, Germany). FIX inhibitor was assayed on the baseline sample, according to Nijmegen method. FIX genotype was performed on whole blood samples at Haemophilia Centre of Castelfranco Veneto by means of Conformation Sensitive Gel Electrophoresis (CSGE) [9], a rapid non-radioactive heteroduplex-based method, followed by sequencing of the purified gene fragment suspected to contain the molecular defect. Comparison with the wild type is performed using the BLAST software, which is developed and maintained by a group at the National Centre for Biotechnology Information (NCBI;www.ncbi.nlm.nih.gov).

The study was approved by the Ethical Committee of Coordinator Centre and by each of other HCC (EudraCT n. 2008-006465-85). It was planned to enrol about $50 \mathrm{HB}$ patients, but unfortunately, many patients denied undergoing such demanding protocol. We decided to stop the study after 5 years when only 17 patients agreed to participate to this study.

\section{Pharmacokinetic analysis}

PK analysis was conducted by means of Phoenix WinNonlin 6.3 software (Certara USA Inc., Princeton, NJ, USA). Non-compartmental analysis (NCA), oneand two-compartment methods (OCM and TCM) were used to evaluate the behaviour of infused Nona$\operatorname{cog}$ alpha and to compare these outcomes with FIX genotype of patients. The baseline FIX:C concentration has been subtracted from each postinfusion concentration of PK.

The parameters reported on Table 1 (Elimination rate constant, IVR, Terminal Half-life, AUC, Clearance, MRT, and Volume of distributions) have been calculated by means of NCA. In addition, OCM and TCM provided K1-0, i.e. the elimination rate constant from central compartment and the corresponding halflife (K1-0_HL). TCM also provided the alpha elimination rate constant associated with the distribution phase and beta elimination rate constant associated with the elimination phase and their respective halflives. In order to evaluate which compartmental model 
Table 1. Non-Compartment and Two-Compartment Analysis of Nonacog alpha pharmacokinetics.

\begin{tabular}{|c|c|c|c|}
\hline Non-compartment analysis & Unit & Mean & $1 \mathrm{SD}$ \\
\hline $\begin{array}{l}\text { Incremental In Vivo } \\
\text { Recovery }\end{array}$ & $\begin{array}{l}\mathrm{IU} \mathrm{dL}-1 \\
\mathrm{IU}^{-1} \\
\mathrm{~kg}^{-1}\end{array}$ & 1.21 & 0.31 \\
\hline Squared $\mathrm{R}$ adjusted & $R^{2}$ & 0.977535 & 0.02166 \\
\hline Elimination constant rate & $1 \mathrm{~h}^{-1}$ & 0.033224 & 0.030618 \\
\hline Terminal Half-life & Hours & 29.81 & 13.22987 \\
\hline Peak & $\mathrm{U} \mathrm{dL}^{-1}$ & 57.52 & 14.9 \\
\hline Trough & $\mathrm{U} \mathrm{dL}^{-1}$ & 7.71 & 4.15 \\
\hline AUC observed, last point & $\mathrm{U}^{*} \mathrm{~h} \mathrm{dL}^{-1}$ & 1442.58 & 426.33 \\
\hline AUC observed, infinity & $\mathrm{U}^{*} \mathrm{~h} \mathrm{dL}^{-1}$ & 1835.78 & 672.12 \\
\hline Clearance & $\mathrm{mL} \mathrm{h}^{-1}$ & 228.30 & 147.0 \\
\hline Clearance normalized by BW & $\mathrm{mL} \mathrm{h}^{-1}$ & 3.01 & 1.35 \\
\hline MRT observed, last point & Hours & 23.76 & 3.51 \\
\hline MRT observed, infinity & Hours & 42.12 & 15.07 \\
\hline Half-life according to MRT & Hours & 29.19 & 10.44 \\
\hline $\begin{array}{l}\text { Volume of distribution }(\mathrm{Vd}) \\
\text { according to terminal } \\
\text { half-life, normalized by BW }\end{array}$ & $\mathrm{mL} \mathrm{kg}^{-1}$ & 110.46 & 30.78 \\
\hline $\begin{array}{l}\text { Volume of distribution (Vss) } \\
\text { at steady state, } \\
\text { normalized by BW }\end{array}$ & $\mathrm{mL} \mathrm{kg}^{-1}$ & 111.1 & 22.66 \\
\hline \multicolumn{4}{|l|}{ Two-compartment model } \\
\hline AUC & $\mathrm{U}^{*} \mathrm{~h} \mathrm{dL}^{-1}$ & 1950.15 & 649.50 \\
\hline K10 Half-life & Hours & 22.30 & 8.04 \\
\hline Alpha, Distribution Half-life & Hours & 4.50 & 4.43 \\
\hline Beta, Elimination Half-life & Hours & 38.35 & 23.10 \\
\hline Cmax & $\mathrm{IU} \mathrm{dL}^{-1}$ & 63.52 & 21.11 \\
\hline Clearance & $\mathrm{mL} \mathrm{h}^{-1}$ & 196 & 69 \\
\hline $\begin{array}{l}\text { Clearance normalized } \\
\text { by BW }\end{array}$ & $\mathrm{mL} \mathrm{h}^{-1}$ & 2.69 & 0.89 \\
\hline MRT & Hours & 49.58 & 23.71 \\
\hline Vss normalized by BW & $\mathrm{mL} \mathrm{kg}^{-1}$ & 121.59 & 56.09 \\
\hline
\end{tabular}

fits better the PK data, we calculated the sum of squared residuals (SSR) according to OCM and TCM. We assumed that the model best fitting the observed data was that with the smaller SSR. Incremental recovery was calculated as ratio between the FIX:C peak (IU dL ${ }^{-1}$ ) and dose $\mathrm{kg}^{-1}$ (IU kg${ }^{-1}$ ), according to Prowse [10].

\section{Results}

The means and 1 SD of each PK outcomes are reported on Tables 1 and 2. The average age of patients was 34.5 years (range: 15.4-60.6 years), and weight average was $74.0 \mathrm{~kg}$ (range: $50.0-110.0 \mathrm{~kg}$ ). The average FIX:C at baseline was $1.2 \mathrm{IU} \mathrm{dL}^{-1}$ (range: $0.1-5.1 \mathrm{IU} \mathrm{dL}^{-1}$ ) and FIX inhibitor was $0.04 \mathrm{BU} \mathrm{mL}^{-1}$ (range: $0.00-0.10 \mathrm{BU} \mathrm{mL}^{-1}$ ). Table 1 reports the outcomes of PK analysis, as far as NCA and TCM methods are concerned. A very good correlation between times and rFIX concentration has been shown by the high values of adjusted $R^{2}$, according to NCA. This is the reason to evaluate the elimination constant rate and terminal half-life by NCA; the linear regression analysis was performed by WinNonlin on about all postinfusion points (generally six out of seven). The average terminal half-life resulted near $30 \mathrm{~h}$, but in seven out of 17 patients was longer than
$30 \mathrm{~h}$. In addition, OCM and TCM confirmed these values, both MRT as well as HL, even though the HL according to the elimination rate from the central plasma compartment (K1-0 HL) of both models were lower, 20.64 and $22.30 \mathrm{~h}$ respectively. Nevertheless, the beta elimination HL by TCM (38.35 h) was very similar to MRTinf of NCA (42.12 h). Very long MRT resulted also by TCM, i.e. $49.58 \mathrm{~h}$. The trough level of rFIX at $72 \mathrm{~h}$ was still high; mean $7.7 \mathrm{IU} \mathrm{dL}^{-1}$ (3$\left.15 \mathrm{IU} \mathrm{dL}^{-1}\right)$. This observation has been confirmed by the high percentage $(18.6 \%)$ of extrapolated AUC to infinity. The clearance and volume of distribution at steady state of Nanacog alpha normalized by patients' body weight resulted $3.09 \mathrm{~mL} \mathrm{~h}^{-1} \mathrm{~kg}^{-1}$ and $111.1 \mathrm{~mL} \mathrm{~kg}^{-1}$ respectively. A paired t Student test comparing the outcomes of OCM and TCM showed statistically significant difference $(P<0.001)$ between the two models, as far as AUC, AUMC, Cmax, Cl, MRT and Vss are concerned (data not shown). The statistically significant smaller SSR achieved by TCM with respect to OCM (45.26 vs. 143.23, $P<0.001)$ shows that TCM fits the Nonacog alpha PK data better than OCM. The range of incremental IVR was $0.89-2.23 \mathrm{IU} \mathrm{dL}^{-1} \mathrm{IU}^{-1} \mathrm{~kg}^{-1}$; the mean being $1.22 \mathrm{IU}^{-1} \mathrm{dL}^{-1} \mathrm{IU}^{-1} \mathrm{~kg}^{-1}$

$\left(1.14 \mathrm{IU}^{-1} \mathrm{dL}^{-1} \mathrm{IU}^{-1} \mathrm{~kg}^{-1}\right.$, short of the highest value).

All genotypes observed in our cohort were classified into three groups: 1 - Small base deletion (SBD, $n=2) ; 2$ - Missense mutation (MM, $n=10) ; 3$ Nonsense mutation (NM, $n=3$ ). Due to the small size of these groups, we reported in Table 2 only the ranges of each PK outcome observed. According to NCA, both patients of the first group displayed the shortest terminal half-life or MRT, and the higher clearance with respect to the all other patients. On the contrary, the longest terminal half-life and the smaller clearance was observed in the three patients of group 3 , even though a partial overlap with patients of group 2 was reported. Cmax, IVR, AUC, and Vss were randomly distributed among the three groups. Even though TCM resulted more accurate than OCM because of its definitively reduced SSR (data not shown), beta HL, clearance and MRT according to TCM did show less significant clustering of outcomes within the genotypes as observed by NCA. A summary of these data are reported in Fig. 1. Among the outcomes of TCM, a good correlation has been observed between MRT and beta HL $\left(R^{2}=0.97\right.$, $P<0.01)$, as well as between terminal half-life and MRT evaluated by NCA $\left(R^{2}=0.61, P<0.02\right)$. Both Clearance from NCA and TCM were well correlated $\left(R^{2}=0.80, P<0.01\right)$. On the contrary, no correlations have been found in our study between MRT by NCA and by TCM, neither between IVR and Vss nor between terminal half-life by NCA and beta HL by TCM. 


\section{Discussion}

The figure of IVR is a bit higher than that, $0.78 \pm 0.24$ or $0.86 \pm 0.21 \mathrm{IU}^{-1} \mathrm{dL}^{-1} \mathrm{IU}^{-1} \mathrm{~kg}^{-1}$, reported by first studies [1,2], confirmed also after the Nonacog alpha reformulation of diluent $(0.73 \pm 0.20$ $\mathrm{IU}^{-1} \mathrm{dL}^{-1} \mathrm{IU}^{-1} \mathrm{~kg}^{-1}$ ) [6]. Very recently, higher values of IVR of Nonacog alpha, $0.93 \pm 0.31 \mathrm{IU}^{-1}$ $\mathrm{dL}^{-1} \mathrm{IU}^{-1} \mathrm{~kg}^{-1}$, have been reported by L.A. Valentino [11] and a very similar figure to our IVR by T. Andreeva at ASH Annual Meeting 2015: $1.24 \pm 0.32$ $\mathrm{IU}^{-1} \mathrm{dL}^{-1} \mathrm{IU}^{-1} \mathrm{~kg}^{-1}$ [12]. These changes of Nonacog alpha concentrate IVR during the last years might reflect some changes in the manufacturing procedures or of the labelled potency.

An accurate and comprehensive PK analysis according to three methods (NCA, OCM and TCM) was able to characterize the Nonacog alpha in vivo behaviour better than in previous studies. The timing of sample collection is crucial to achieve outcomes that are more reliable. Even though the ISTH recommendation stated for PK of FIX concentrate that the last point could be the $72 \mathrm{~h}$, more than $18 \%$ of AUC was lost in this study, due to very high trough of decay curve. Unfortunately, none PK studies respected the golden rule of pharmacokinetics that sample collection must be continued up to the baseline is again achieved. On the other hand, it is difficult to forecast a priori the time of next achieved baseline. Also in our study, the range of trough was high. In all recent published comparative studies between new long-acting rFIX and Nonacog alpha, the sampling time of the comparators, pdFIX and Nonacog alpha, has been stopped too early, generally at $48 \mathrm{~h}$ after the end of infusion [13-15]. This is the reason why lower values of AUC, higher clearance and shorter half-life have been reported with respect to the outcomes of our study. On the contrary, when the sampling time was

Table 2. PK outcomes by NCA and TCM according to HB patients' genotype.

\begin{tabular}{|c|c|c|c|c|c|c|c|c|c|}
\hline & \multicolumn{6}{|c|}{ Non-compartment analysis } & \multicolumn{3}{|c|}{ Two-compartment method } \\
\hline & $\begin{array}{c}\text { Terminal HL } \\
\text { Hours }\end{array}$ & $\begin{array}{l}\text { Cmax } \\
\text { IU dL }\end{array}$ & $\begin{array}{c}\text { AUC } \\
\mathrm{U}^{*} \mathrm{~h} \mathrm{dl}^{-1}\end{array}$ & $\begin{array}{l}\text { MRT } \\
\text { Hours }\end{array}$ & $\mathrm{mL} \mathrm{h}^{-1} \mathrm{~kg}^{-1}$ & $\begin{array}{c}\text { Vss } \\
\mathrm{mL} \mathrm{kg}^{-1}\end{array}$ & $\begin{array}{l}\text { Beta_HL } \\
\text { Hours }\end{array}$ & $\mathrm{mL} \mathrm{h}^{-1} \mathrm{~kg}^{-1}$ & $\begin{array}{l}\text { MRT } \\
\text { Hours }\end{array}$ \\
\hline Min & 5.02 & 47.7 & 673 & 15.57 & 4.17 & 76 & 19.04 & 2.64 & 27.14 \\
\hline $\operatorname{Max}$ & 9.69 & 59.3 & 1227 & 18.08 & 6.73 & 106 & 30.14 & 3.22 & 41.72 \\
\hline \multicolumn{10}{|c|}{ Missense mutation $n=10$} \\
\hline Min & 14.17 & 41.2 & 812 & 17.69 & 1.51 & 75 & 20.01 & 1.26 & 26.34 \\
\hline Max & 42.20 & 106.3 & 2393 & 27.27 & 5.22 & 134 & 105.65 & 3.25 & 108.85 \\
\hline \multicolumn{10}{|c|}{ Nonsense mutation $n=3$} \\
\hline Min & 33.84 & 52.0 & 1420 & 25.17 & 1.63 & 98 & 34.60 & 1.07 & 46.73 \\
\hline Max & 57.45 & 64.2 & 2060 & 26.76 & 2.76 & 162 & 78.89 & 2.08 & 95.92 \\
\hline
\end{tabular}
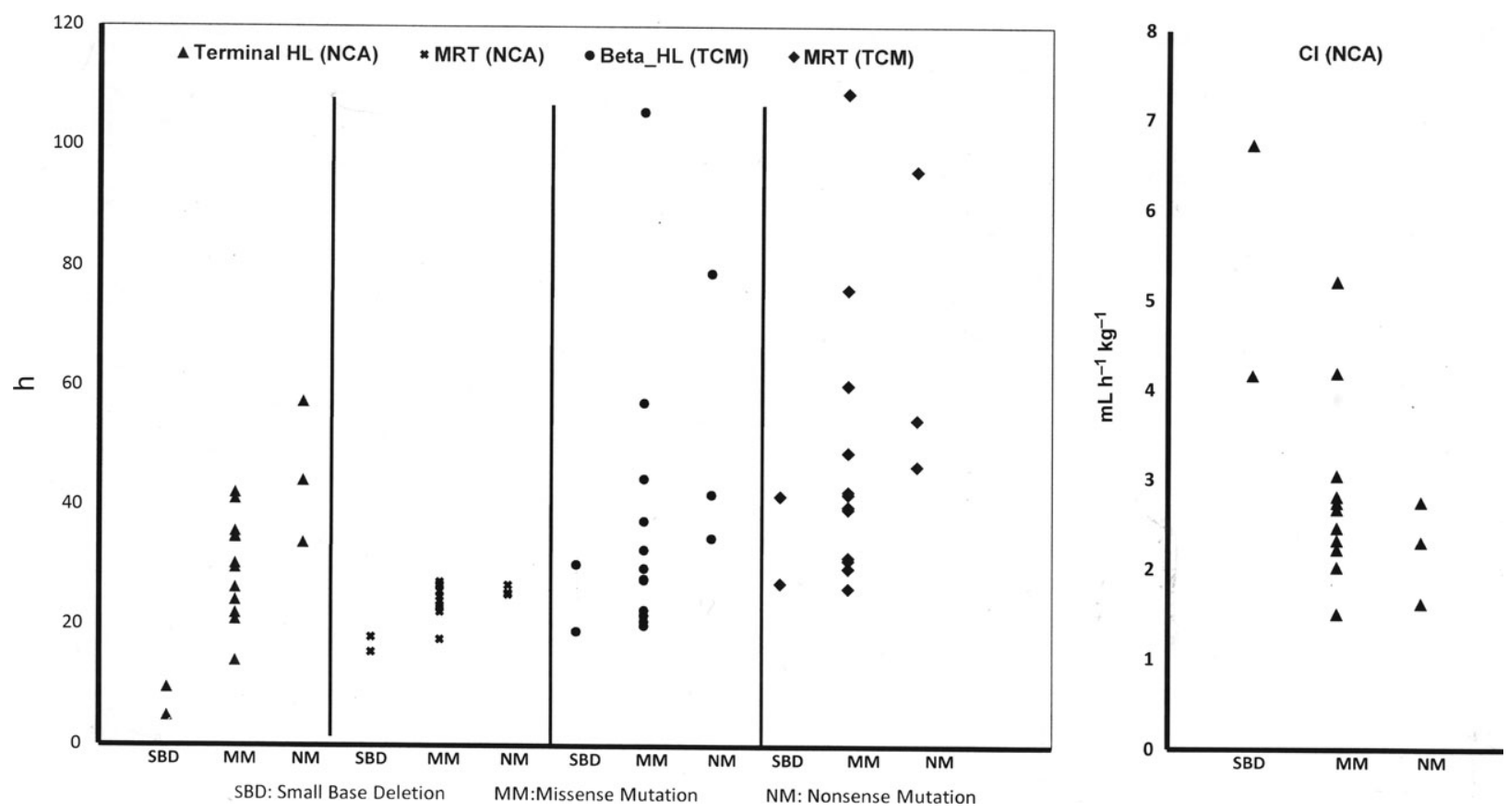

Fig. 1. Terminal half-life, MRT and clearance according to NCA; MRT and beta half-life according to TCM. 
prolonged up to $72 \mathrm{~h}$ [16-18], the PK outcomes resulted very similar to ours. The average values of all clearances observed in our study resulted 50\% less than those reported in previous comparative studies of N9-GP or rFIX-FP [14,15] because of different AUC. The clearance observed in this study is similar to that of rFIX-Fc [17]. In addition, the Vss was quite small compared with the large Vss of rFIX-Fc, whose mean value was $250 \mathrm{~mL} \mathrm{~kg}^{-1}$ [19]. The large Vss of rFIXFc is probably due to its uptake by Fc receptor bearing cells.

Even though the size of this study is small, due to the difficulties to enrol HB patients in such a demanding protocol, the relationship between genotype and PK outcomes resulted quite interesting. Patients with SBD and with NM showed an opposite behaviour as far as terminal half-life, clearance, MRT and beta half-life were concerned. Outcomes of NCA seems to be able to better discriminate differences among the three groups of genotype. NCA is confirmed to be the most robust and reliable method of analysis of PK data because it is not affected by variance and errors of fitting procedure of all compartment methods. Model-independent method is more reliable than compartment methods both for FVIII and FIX concentrates, as our working group stated many years ago [20,21]. OCM resulted definitely less accurate than TCM because of its higher SSR. TCM seems to fit better the PK data of rFIX, as also recently reported [22]. We would like to recommend that the choice of the best fitting compartment method should be based on SSR.

\section{Conclusion}

The analysis of single-dose PK multicentre study showed different results from those previously published because of different sampling times. Even though the last sample was collected $72 \mathrm{~h}$ after the end of infusion, $18 \%$ of Nonacog Alpha AUC was lost. Clearance resulted about $50 \%$ less of that reported in studies where the sampling was stopped at $48 \mathrm{~h}$. Higher values of terminal half-life, MRT and beta half-life have been observed by NCA or TCM. TCM fitted very well the PK data as shown by the very low SSR. Two patients with SBD displayed the shortest terminal half-life and the higher clearance than those with MM or NM. Due to the relationship between FIX genotype and clearance, tailored prophylaxis of $\mathrm{HB}$ patients can be partially predicted by genotyping.

\section{Acknowledgements}

\begin{abstract}
We thank Pfizer for having supported the laboratory test expenses with an unrestricted grant. M. Morfini performed the PK analysis and statistical evaluation of outcomes, A. Dragani, P. Radossi, G. Di Minno, M.G. Mazzucconi, G. Rossetti, G. Barillari, M. Napolitano, S. Linari and A. Natale performed Nonacog Alpha pharmacokinetic studies, E. Paladino performed the FIX assays. D. Belvini and R. Salviato performed the FIX genotyping, M Morfini and G. Tagariello wrote the article. MM acted as paid consultant to Bayer, Baxter, Novo Nordisk, Pfizer Advisory Boards and received a fee as invited speaker at CSL Behring Symposia and Biotest, Bayer and Novo Nordisk. He received also research grants from Bayer, Pfizer and Baxter. GDM acted as paid speaker for Boehringer-Ingelheim, Sanofi-Aventis, Bayer, Novo Nordisk, Pfizer, Biotest and Grifols, and as consultant for BoehringerIngelheim, Eli-Lilly, Sanofi-Aventis, Bayer, CSL Behring, Novo Nordisk, Pfizer, Biotest and Grifols. MGM took part as paid speaker for Amgen, Glaxo-Novartis, Shire, Baxter, Novo Nordisk, Bayer, Pfizer, Kedrion, and to the Novo Nordisk Advisory Board. G.T. received reimbursement for attending Symposia from Novo Nordisk, Baxter and Pfizer.
\end{abstract}

\section{Disclosures}

The authors stated that they had no interests which might be perceived as posing a conflict or bias.

\section{References}

1 White GC 2nd, Beebe A, Nielsen B. Recombinant factor IX. Thromb Haemost 1997; 78: 261-5.

2 Ewenstein BM, Joist JH, Shapiro AD et al. Pharmacokinetic analysis of plasma-derived and recombinant F IX concentrates in previously treated patients with moderate or severe hemophilia B. Transfusion 2002; 42: 190-7.

3 Roth DA, Kessler CM, Pasi KJ, Rup B, Courter SG, Tubridy KL, Recombinant Factor IX Study Group. Human recombinant factor IX: safety and efficacy studies in hemophilia B patients previously treated with plasma-derived factor IX concentrates. Blood 2001; 98: 3600-6.

4 White GC 2nd, Shapiro A, Ragni M et al. Clinical evaluation of recombinant factor IX. Semin Hematol 1998; 35(Suppl. 2): 33-8.

5 Björkman S, Shapiro AD, Berntorp E. Pharmacokinetics of recombinant factor IX in relation to age of the patient: implications for dosing in prophylaxis. Haemophilia 2001; 7: 133-9.

6 Lambert T, Recht M, Valentino LA et al. Reformulated BeneFix: efficacy and safety in previously treated patients with moderately severe to severe haemophilia B. Haemophilia 2007; 13: 233-43 Erratum in: Haemophilia 2007; 13: 450.

7 Morfini M, Lee M, Messori A. The design and analysis of half-life and recovery studies for factor VIII and factor IX. Factor VIII/Factor IX Scientific and Standardization Committee of the International Society on Thrombosis and Haemostasis. Thromb Haemost 1991; 66: 384-6.

8 Lee M, Morfini M, Schulman S, Ingerslev J and the Factor VIII/Factor IX Scientific and Standardization Committee of the International Society on Thrombosis and Haemostasis. Available at: https://c.ymcdn.com/sites/ www.isth.org/resource/group/d4a6f49a-f4ec -450f-9e0f-7be9f0c2ab2e/official_communi
cations/fviiipharmaco.pdf?hhSearchTerms= $\% 22$ morfini\%22. Accessed January 2, 2016.

9 Belvini D, Salviato R, Radossi $\mathrm{P}$ et al., AICE HB Study Group. Molecular genotyping of the Italian cohort of patients with hemophilia B. Haematologica 2005; 90: 635-42.

10 Prowse CV. In vivo recovery of factor VIII following transfusion: a survey of recent data and publications to assess the influence of standards used for potency assignment. On behalf of the Subcommittee on Factor VIII and IX of the Scientific and Standardization Committee of the ISTH. Thromb Haemost 1995; 74: 1191-6.

11 Valentino LA, Rusen L, Elezovic I, Smith LM, Korth-Bradley JM, Rendo P. Multicentre, randomized, open-label study of ondemand treatment with two prophylaxis regimens of recombinant coagulation factor IX in haemophilia B subjects. Haemophilia 2014; 20: 398-406. 


\section{M. MORFINI et al.}

12 Andreeva T, Zorenko VY, Davydkin I et al. Safety and efficacy of New Nonacog Alfa Drug (Innonafactor) in Prophylactic treatment in patients with severe and moderate Hemophilia B. Blood 2015, 126, Abstract n. 3532.

13 Alamelu J, Bevan D, Sorensen B, Rangarajan S. Pharmacokinetic and pharmacodynamic properties of plasma-derived vs. recombinant factor IX in patients with haemophilia B: a prospective crossover study. $J$ Thromb Haemost 2014; 12: 2044-8.

14 Negrier C, Knobe K, Tiede A, Giangrande P, Møss J. Enhanced pharmacokinetic properties of a glycoPEGylated recombinant factor IX: a first human dose trial in patients with hemophilia B. Blood 2011; 118: 2695-701.

15 Santagostino E, Negrier C, Klamroth R et al. Safety and pharmacokinetics of a novel recombinant fusion protein linking coagulation factor IX with albumin (rIX-
FP) in hemophilia B patients. Blood 2012; 120: 2405-11.

16 Martinowitz U, Shapiro A, Quon DV et al. Pharmacokinetic properties of IB1001, an investigational recombinant factor IX, in patients with haemophilia B: repeat pharmacokinetic evaluation and sialylation analysis. Haemophilia 2012; 18: 881-7.

17 Lissitchkov T, Matysiak M, Zavilska K et al. Head-to-head comparison of the pharmacokinetic profiles of a high-purity factor IX concentrate (AlphaNine ${ }^{\circledR}$ ) and a recombinant factor IX $\left(\right.$ BeneFIX $\left.^{\circledR}\right)$ in patients with severe haemophilia B. Haemophilia 2013; 19: 674-8.

18 Aznar JA, Cabrera N, Matysiak M et al. Pharmacokinetic study of a high-purity factor IX concentrate (Factor IX Grifols) with a 6-month follow up in previously treated patients with severe haemophilia B. Haemophilia 2009; 15: 1243-8.
19 Shapiro AD, Ragni MV, Valentino LA et al. Recombinant factor IX-Fc fusion protein $(\mathrm{rFIXFc})$ demonstrates safety and prolonged activity in a phase $1 / 2$ a study in hemophilia B patients. Blood 2012; 119: 666-72.

20 Longo G, Matucci M, Messori A, Morfini M, Rossi-Ferrini P. Pharmacokinetics of a new heat-treated concentrate of factor VIII estimated by model-independent methods. Thromb Res 1986; 42: 471-6.

21 Longo G, Cinotti S, Filimberti E et al. Single-dose pharmacokinetics of factor IX evaluated by model-independent methods. Eur J Haematol 1987; 39: 426-33.

22 Collins PW, Møss J, Knobe K, Groth A, Colberg T, Watson E. Population pharmacokinetic modeling for dose setting of nonacog beta pegol (N9-GP), a glycoPEGylated recombinant factor IX. J Thromb Haemost 2012; 10: 2305-12.

\section{Appendix}

Contributors of Benefit Study group: Dr. Donata Belvini and Dr. Roberta Salviato, Transfusion Service, Haemophilia Centre, Castelfranco Veneto
Hospital, Dr. Silvia Linari, Agency for Haemophilia, University Hospital of Florence, Dr. Annalisa Natale, Haemophilia \& Thrombosis Centre, Haematology Department, Regional Hospital, Pescara, Italy. 